\title{
SCANAM method for the assessment of industrial noise
}

\author{
Aleksandar Zunjic* \\ University of Belgrade, Faculty of Mechanical Engineering, Kraljice Marije 16, 11000 Belgrade, Serbia
}

\begin{abstract}
Work in an industrial environment is often associated with high levels of noise. For measuring and evaluating of noise levels, sometimes it is necessary to use several instruments. These instruments can be very expensive and require qualified personnel. Medium and small-sized firms often lack sufficient financial resources to ensure continuous measurement and assessment of noise levels. For this reason, a new SCANAM method for assessing the levels of noise is designed. This method involves the use of cheap and accurate sound level meter and specially designed software for assessing the effects of noise. Application of this method is very simple. Based on a noise sample in duration of eight hours, the accuracy of the SCANAM method was confirmed. Application of this method is significantly cheaper than an approach based on the use of expensive multi-function devices, or separate devices for measuring and assessing noise at the workplace.
\end{abstract}

Keywords: SCANAM method, noise, noise assessment

\section{Introduction}

Workers in the industry are mostly exposed to the high noise levels. In an industrial environment, noise levels in some cases do not drop below the allowed limit. As a result, there is frequent appearance of professional disorders of workers in industry caused by high noise levels, whereby sometime it is also endangered the safety of workers. For this reason, in almost all countries of the world there are appropriate laws and standards that regulate the values that are obligatory for determination, in order to evaluate detrimental effects of noise and level of the hazard.

However, in industrial enterprises, especially those of small and medium size, often there are no employed persons who would be exclusively dealt with issues of safety and health at work. For this reason, in such companies the noise is rarely an object of measurement and assessment, which as a consequence may have impairment of the sense of hearing, reduction of work efficiency and opportunities for communication, as well as endangering the safety of workers. Managements of such firms often do not have a fund for purchasing of expensive measure- ment equipment, such they are multifunctional sound level meters, noise dosimeters and so on. In addition, managements of such firms do not have enough time to seriously address this problem. To the solution of described problems, the author of this paper presents a new SCANAM method (Simple Cheap Accurate Noise Assessment Method) for the evaluation of effects of noise in an industrial environment.

\section{Method}

The SCANAM method involves the usage of cheep and precise sound level meter, as well as specially designed software application for the assessment of existing nose levels. This new software enables determination of appropriate values for the evaluation of noise, which are in an international use, such as equivalent sound level, noise dose and time weighted average (TWA).

Equivalent sound level $\mathrm{L}_{\mathrm{eq}}$ is equal to the soundpressure level of a constant noise, which over a given time period, transmits to the receiver the same amount of acoustic energy as the actual time-varying

\footnotetext{
*Address for correspondence: Aleksandar Zunjic, Ph.D., Assoc. Prof., Department of Industrial Engineering, Faculty of Mechanical Engineering, University of Belgrade, Kraljice Marije 16, 11000 Belgrade, Serbia, Tel.: +381642537486; E-mail: azunjic@mas.bg.ac.rs
} 
sound [2]. Noise dose is the main concept in OSHA requirements. A partial dose is the ratio between the time actually spent at the sound level and the maximum permissible time at the sound level. The total noise dose is equal to the sum of the partial doses. Exposures to noise levels that are less than $80 \mathrm{dBA}$ are ignored in calculating doses [3]. TWA is the nose level that would produce a given noise dose if a worker were exposed to that sound level continuously over an 8-h workday [3].

The essence of the method consists in the application of accurate and cheap sound level meter, with which noise levels are initially measured, at the observed workplace. After that, the measured values are transferred into the software application, which automatically calculates values of the equivalent sound level, the noise dose and the TWA. This software application also automatically generates the assessment of noise levels in relation to the aforementioned values. In this way, we can easy get information on whether the noise at a workplace satisfies in relation to the calculated values and criteria for evaluating the level of noise.

Accordingly, the SCANAM method consists of several following steps:

1. By using of a precise and low-cost sound level meter on the selected workplace (or for selected employees) it is necessary to measure noise levels. It is recommended to conduct reading and recording of noise levels every $5 \mathrm{~s}$ or $10 \mathrm{~s}$. Longer total measurement time implies a more realistic picture of actual conditions. The most convenient is to perform noise levels recording during a work shift. Measured values should record in a table previously prepared on a paper, if a sound level meter does not have its own memory.

2. Measured values of noise levels should be transferred into the SCANAM program. Besides the noise levels, into the program should enter two more data. These are: the length of the time interval (in seconds) between successive readings of noise levels and the total time of measurement of noise levels in seconds.

3. After completing the first two steps, the program automatically calculates the equivalent sound level, noise dose and TWA. Based on each of these three values, the program momentarily generates the assessment of effects of noise. In addition, in separate windows, the program generates the histogram of the noise and the cumulative diagram of noise level changes over the time.
The histogram of noise is a graphical representation of noise level changes, in relation to the time representation of the observed noise level in percentages [4]. Construction of the histogram of noise changes over the time is particularly important for the assessment of the environment in which noise exists, because from the histogram of noise can be determined the percentage of time in which a particular interval of noise existed. Cumulative diagram of noise level changes over the time is also important for the assessment of exposure to the noise. From the cumulative diagram can be determined the noise levels that are exceeded during a certain period of time, such are, for example, $\mathrm{L}_{10}, \mathrm{~L}_{50}$ and $\mathrm{L}_{90}$.

In order to evaluate the accuracy of the SCANAM method, the noise levels were measured during 8 hours, in a factory for manufacturing of buses. The values of noise levels were recorded every 10 seconds. Handheld sound level meter Bruel \& Kjaer type 2232 was used. The measurement was conducted at the workplace, which contains a press. To determine the equivalent noise level, as an alternative method, the method of Taylor and Lipscomb was used [2]. The noise dose and the TWA were alternatively determined by calculation.

\section{Results}

Based on the implementation of the SCANAM program, following results were obtained:

Equivalent sound level is $97.55 \mathrm{~dB}$

Noise dose is $88.71 \%$

TWA is $89.14 \mathrm{~dB}$.

Assessments based on previous three obtained values, which were generated by the SCANAM program:

"The permitted noise level is exceeded" assessment based on the $\mathrm{L}_{\mathrm{eq}}$ value

"The program for hearing protection is necessary" - assessment based on the noise dose

"The program for hearing protection is necessary" - assessment based on the TWA value.

Fig. 1 shows the histogram of the noise that was generated by the SCANAM program. In Fig. 1 can be noticed that the interval of noise levels from $75 \mathrm{~dB}$ to $80 \mathrm{~dB}$ lasted the longest time. Its time participation was $37.81 \%$ of the total measurement time ( 8 hours). Fig. 2 shows the cumulative diagram of noise level changes over the time, which was generated by the SCANAM program. By means of Fig. 2, in a simple manner, as described in [5] and in the SCANAM 


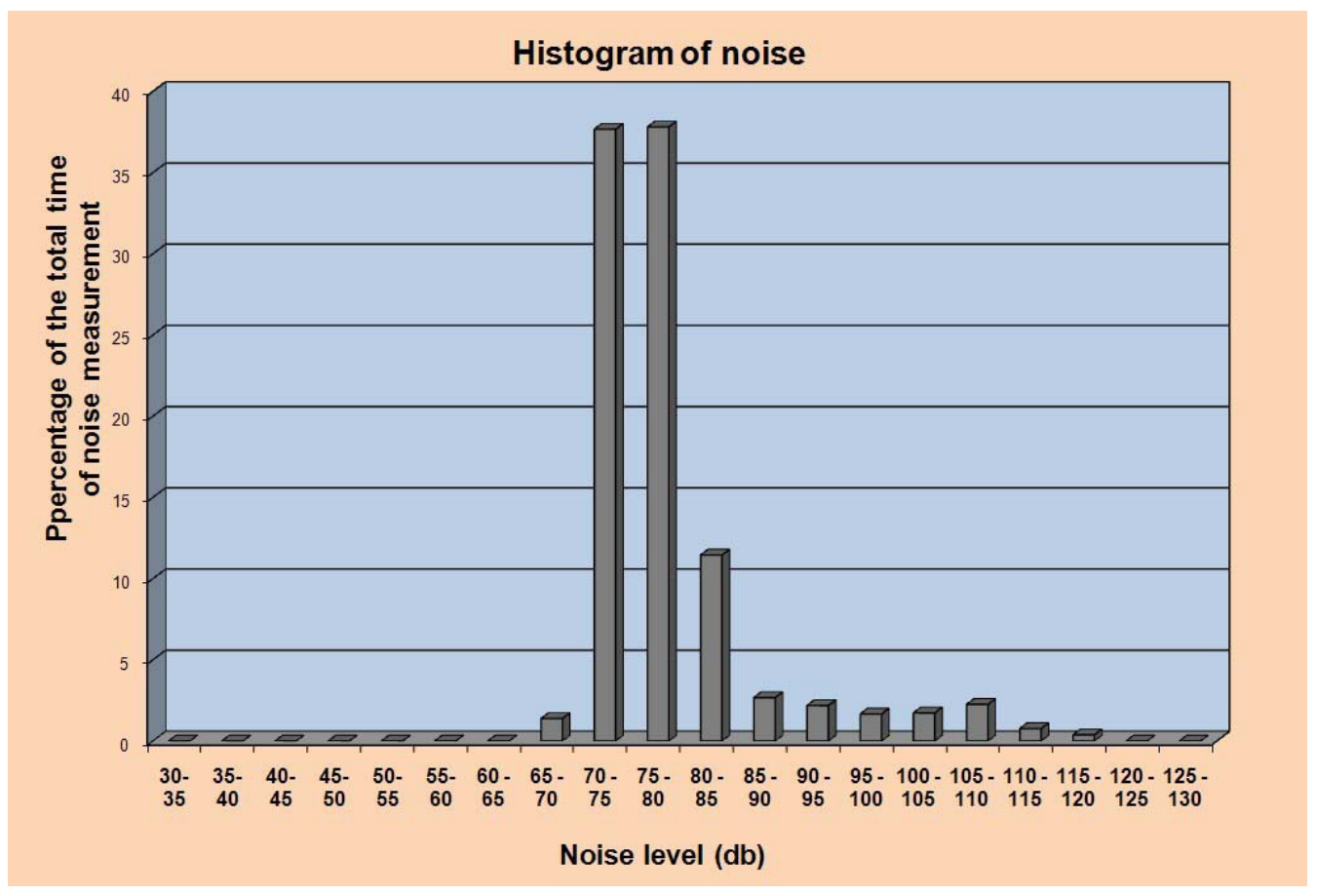

Fig. 1. Histogram of noise that is generated by the SCANAM program.

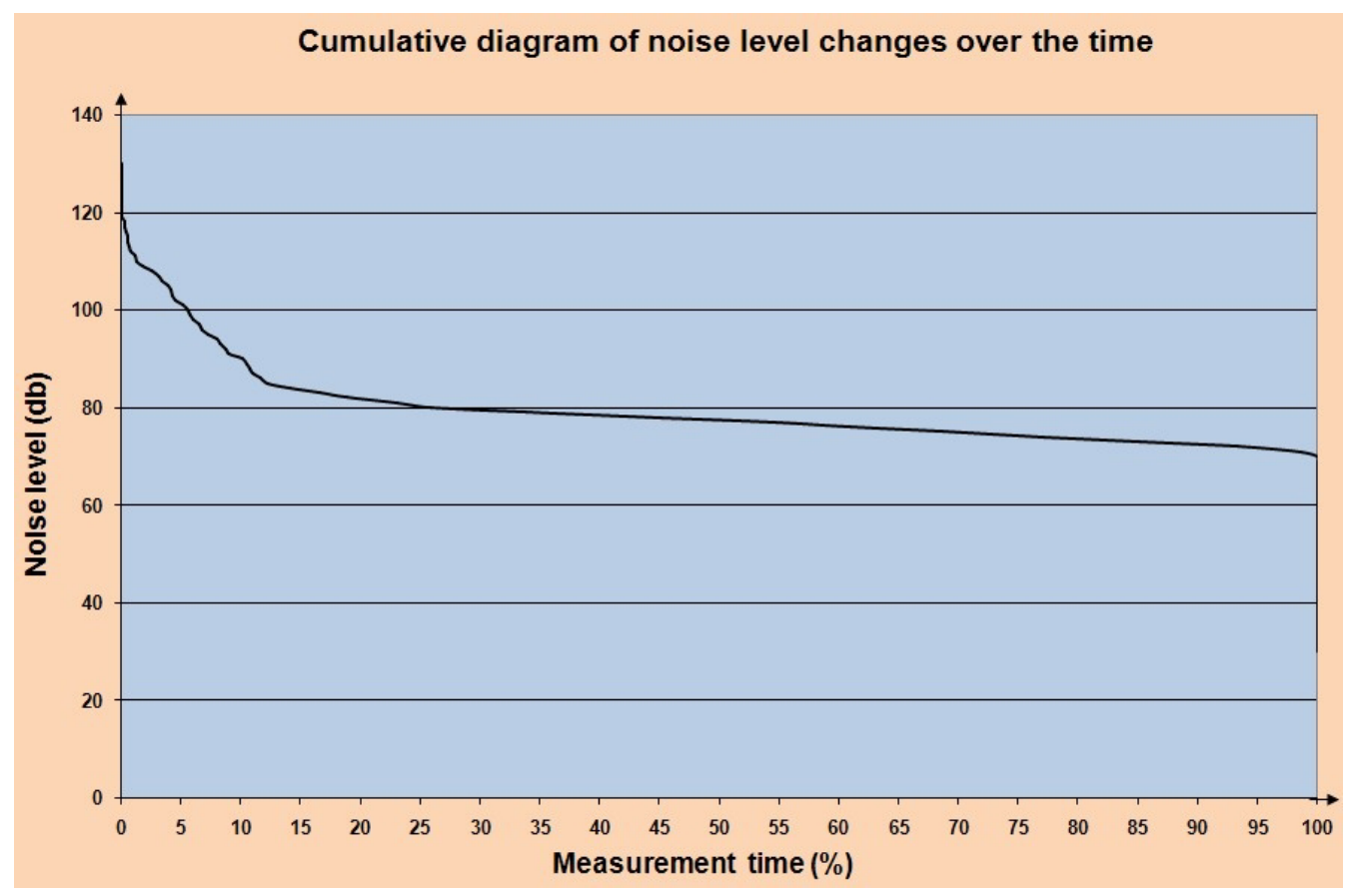

Fig. 2. Cumulative diagram of noise level changes over the time that is generated by the SCANAM program. 
instruction manual, can be determined the noise levels that were prevailed during certain time intervals. In this way, we get that the level $\mathrm{L}_{10}$ equals $90 \mathrm{~dB}$. This means that the noise levels of 90 or more decibels were represented with $10 \%$ of time, when taken into account the entire interval of measurement. Based on the same procedure we get that the level $\mathrm{L}_{50}$ amounts to $77.5 \mathrm{~dB}$, and that the level $\mathrm{L}_{90}$ amounts to $72.5 \mathrm{~dB}$.

The value of the equivalent sound level that is obtained by applying the SCANAM program will be compared with the value for Leq, which is obtained using the method of Taylor and Lipscomb. The method of Taylor and Lipscomb for determining of the equivalent sound level involves several steps. The last step of this procedure is shown in Fig. 3. This figure shows combining of partial Leq values by using of the nomograph, in order to obtain the final value of the $\mathrm{L}_{\text {eq }}$. Fig. 3 shows that the final $\mathrm{L}_{\text {eq }}$ value equals $97.64 \mathrm{~dB}$. The difference between the $\mathrm{L}_{\text {eq }}$ value that is obtained by use of the SCANAM method and the method of Taylor and Lipscomb in this case is only $0.09 \mathrm{~dB}$.

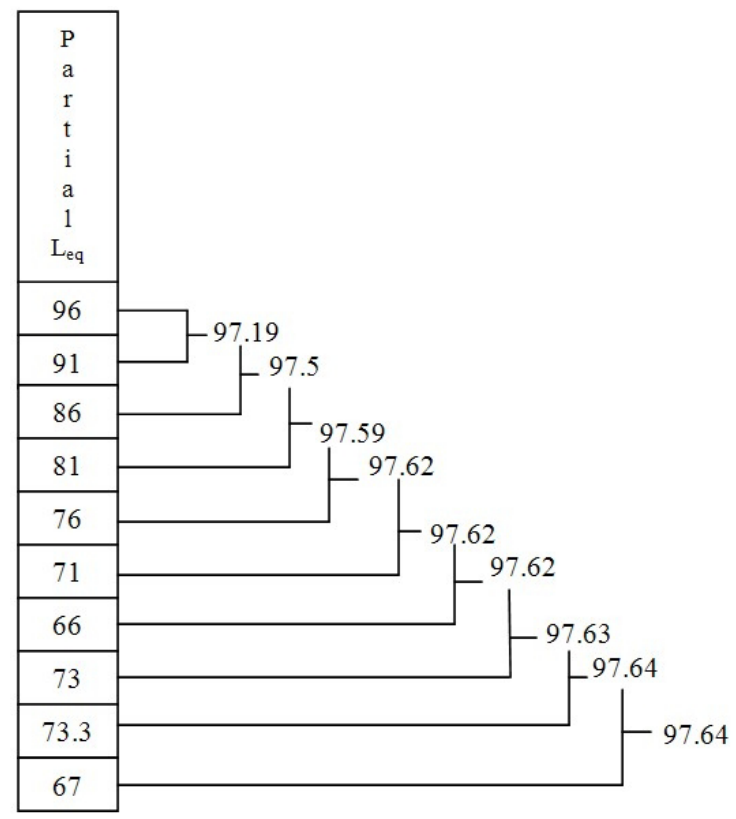

Fig. 3. Combining of partial values of equivalent sound levels according to the method of Taylor and Lipscomb, in order to obtain the final value of equivalent sound level.

The value of the noise dose that is obtained by applying the SCANAM program will be compared with the value for noise dose, which is obtained using the formula. Forty partial noise doses were calculated. The total noise dose $\mathrm{D}$, which was calculated by the formula as a sum of partial doses $D_{i}$, equals $88.3 \%$. The difference between the noise dose that is obtained by use of the SCANAM method and by use of the formula, in this case equals $0.41 \%$.

The value of the TWA that is obtained by applying the SCANAM program will be compared with the value for the TWA, which is obtained using the formula. Substituting the calculated value for D in the formula for TWA, the value for TWA is obtained and it equals in this case $89.1 \mathrm{~dB}$. The difference between the TWA value that is obtained by use of the SCANAM method and by use of the formula, in this case equals $0.04 \mathrm{~dB}$.

Finally, the values of the histogram and the cumulative diagram of noise level changes over the time were also verified. There has been accordance between the values that are represented in diagrams, and those that are obtained through calculations.

Based on the results of the noise measurement, it can be concluded that working conditions at the observed workplace are not appropriate. The main reason for this is because the measures for the reduction of noise levels in this factory are not implemented. It is necessary that the factory management takes appropriate measures of engineering and administrative control, to reduce the noise at the observed workplace.

\section{Discussion}

Criteria for assessment of the effects of noise depend on the regulations and standards that are applied in certain states. The SCANAM version of the program that is presented in this paper uses the criteria for assessing the effects of noise, which are in force in Serbia. These criteria are slightly different from the criteria used by the OSHA. In addition, some criteria for assessing the effects of noise also depend on the type of activities that a worker performs. The criteria that were applied to assess the effects of noise based on the equivalent sound level in this version of the program, especially take into account the workers in the industry who perform physical work, focused on accuracy and periodically monitoring of the environment by use of hearing. Having that in mind, different versions of the program are foreseen, depending on the criteria for the assessment that should be applied. An integral version of the program that includes multiple criteria is also an option. 
Assessment of noise that generates the main part of the program is very concise and contains no further explanation. The reason for this is that the program is primarily intended for users with no previous experience in dealing with noise. Robust explanations for such users potentially would be able to cause a confusion. Fig. 4 shows a part of the main window of the SCANAM program, which refers to the data entering and assessment. However, the user manual additionally describes basic measures to be taken when the criteria for safe work are not satisfied. This primarily refers to situations when the action level is reached $(\mathrm{D}=50 \%)$ or the permissible noise level $(\mathrm{D}=100 \%)$.

Bearing in mind the population of users to which the program is primarily intended, it is necessary that the program is easy to use, efficient, easy for training (if it is necessary), and that the users are satisfied when using it. The most important requirement is to ensure ease of use. The program is designed to be used by people who had no prior experience in working on this type of work, or who have no previous knowledge that is needed. Bearing this in mind, it was necessary to design the interface, which will support the above requirements. For this purpose, 10 Nielsen's heuristics [1] that relates to the usability of the interface were used, for designing of the SCANAM application. Discussion that follows shows the heuristics that were used and the way in which they were applied when designing the SCANAM program.

\subsection{Visibility of system status}

The main goal of this heuristic is to provide explicit information about what is going on, through appropriate feedback within the reasonable time.

There are four main windows (displays). These are: data and assessment, the histogram, the cumulative diagram and the manual. Every window begins with a title or header that describes the screen content. For example, the window that relates to data entry and calculations has two parallel headers: instructions for data entering and results and assessment. In this way, it becomes clear where the user should enter the necessary information and where to expect the results of the analysis.

The only activity that a user needs to perform is the task of data entry. Data entry is performed in the appropriate fields. The location (coordinate) of each field is determined by alphanumeric characters in the horizontal and vertical direction, so that the user can easily determine visually the location of each data. Frame that surrounds the place of data entry additionally contributes to the visual identification of the active field. After entry of a numeric value in the field and activation of the "enter" command, the frame shifts to the next field, providing in such a way the visual feedback after each user activity. Additionally, this form of feedback indicates that the next action on the data entry can be started. Position of the cursor also indicates which field is activated.

Every response of the system is very fast, less than $1 \mathrm{~s}$, in all cases (when the minimum system requirements are satisfied). This response time is appropriate to the user's cognitive processing. High levels of concentration aren't necessary and remembering information is not required. State of the system can be easy determined at any time. This is supported by the appropriate instructions, which appear when the necessary action of a user is missing. The application uses appropriate labels placed at the bottom of the screens, when users need to navigate between screens.

\subsection{Match between the system and the real world}

This heuristic implies that the system should speak the users' language, with words and phrases that are familiar to the user. System-oriented terms should be avoided. Application of real-world conventions, arranging of information in a natural and logical order is the main purpose of this heuristic.

Related and interdependent fields, such as data entering, results and assessment, are arranged in the SCANAM program so that they appear on the same screen. All information on the main screen (data and assessment) are color coded. In order to attract users' attention to the fields for data entry, they are colored red. The program will not release results in the case when the data are not entered. Red color of fields for data entry indicates the location where the omission is made. The fields in which appear results and assessment are colored green. This color also indicates that the user does not need to take any correction in these fields related to data entry. Instructions and explanations are colored yellow.

When an instruction implies a necessary action, the words in a message are consistent with that action. For example, one of the instructions for data entering looks "In the field H12, enter the total time of measurement of noise levels in seconds". All other tasks are also described in terminology familiar to users. Computer jargon is avoided. 


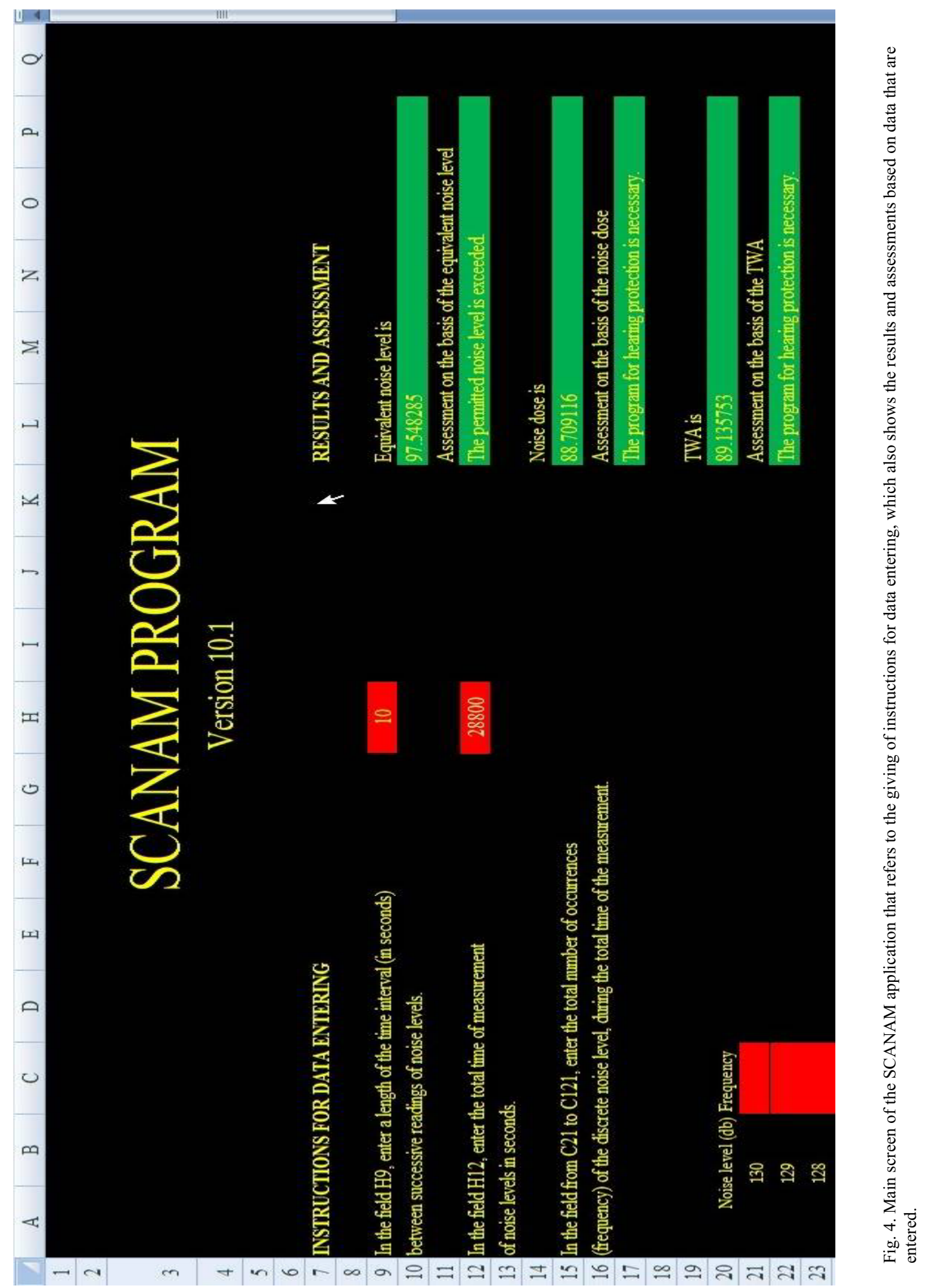




\subsection{User control and freedom}

This heuristic refers to the freedom of selection of sequence of tasks (when appropriate), rather than having the systems perform this for them. Users often choose system functions by mistake. They need a clearly marked "emergency exit" to leave the unwanted state, without having to go through an extended dialogue.

Possibility of activation of unwanted commands is minimized in the SCANAM application. However, if an unwanted command is activated accidentally, it is possible a cancellation of execution of commands that may have drastic consequences (by pressing the cancel button or the x sign). "Undo" command at the level of a single action, such as a data entry, also is supported. For sequence of actions, multiple undo function is also available. Users can move forward and backward between data entry fields.

\subsection{Consistency and standards}

This heuristic suggests that users should not have to wonder whether different words, situations, or actions mean the same thing. Platform conventions should be followed.

Formatting standards has consistently followed in all screens within the application. There are salient visual cues to identify the active window (at the bottom of each SCANAM window). Vertical and horizontal scrolling are possible in each window. More than four colors were not used per window. The same font is used on all windows. User actions are named consistently across all prompts in the application. Method for moving the cursor to the next or previous field is consistent throughout the application.

\subsection{Error prevention}

This heuristic states that a careful designing that prevents a problem is even better than good error messages.

Borders of the fields for data entering in the SCANAM application are clearly marked, which prevents making mistakes of entering data in other fields. Moreover, data entry is disabled in any other field, except in fields that are provided for data entry. Navigation between windows is simple and visible. The program prevents users from making errors whenever possible. For example, the user cannot accidentally delete or change values in the fields provided for the results and assessments.

\subsection{Recognition rather than recall}

The main goal of this heuristic is to minimize the user's memory load by making objects, actions and options visible and easy accessible. The user should not have to remember an instruction. Instructions for use of the system should be visible or easily retrievable whenever appropriate.

All basic instructions about data entering in the SCANAM application are grouped. They are positioned in the upper left side of the main screen, so they are easy accessible to the user. Between text messages (instructions) certain empty space exists, so that they could easily be noticed. Although all the instructions necessary for the operation of the program are contained in the user manual, in order to minimize recall of users, besides all places that are provided for the data entry are precise instructions. Items are grouped into logical zones, and headings are used to distinguish between zones. For example, the results are not separated from the assessment. Immediately next to each result is the assessment based on that result. In this way, information that are associated are easily visible.

\subsection{Flexibility and efficiency of use}

Generally speaking, this heuristic suggests that a system should serve inexperienced, as well as experienced users.

The SCANAM program is primarily intended for users who do not have much experience in connection with measuring and evaluating the effects of noise. However, the program is designed so that it can be used equally efficient for inexperienced and experienced users. The main reason for this is simplicity of use of the program.

\subsection{Aesthetic and minimalist design}

This heuristic suggests that dialogues should not contain information, which is rarely needed or irrelevant. Every additional unit of information in a dialogue competes with the relevant units of information, reducing their relative visibility.

Each instruction (information) in the SCANAM application relates to only one item, which is essential for the work of the program. Each instruction is 
precisely formed and directed to the immediate execution of a task or goal. There are not extensive descriptions that can focus user's attention on unimportant details.

\subsection{Help users recognize, diagnose, and recover from errors}

This heuristic indicates that an error message should be expressed in the plain language, precisely reveal the problem and constructively suggest a solution.

It is necessary to point out that the possibility of making mistakes when using the SCANAM program is reduced to a minimum. However, if the user makes a mistake, a message appears on the error. Error messages are written in plain language, and they do not contain the code. They use a consistent grammatical style, form and terminology.

\subsection{Help and documentation}

This heuristic suggests that information in the manual should be easy to search, focused on the user's task, contain a list of concrete steps that should be carried out and to be concise.

The SCANAM program is designed to be intuitive, so that user can perform the entire task about input of data and reading of the results. User manual an evaluator can use if he wants to check whether he understood well the manner of functioning of the program. However, the guide also provides information for absolute beginners. This manual contains even basic information on noise, on noise measurement procedure and the recording of measured values. The manual also contains basic information about the dimensions and values that represent the results of measurements, on assessment of hazards of noise based on these results and the actions to be taken to eliminate the harmful consequences.

\section{Conclusion}

Difference between the values of the equivalent noise level that was obtained by the SCANAM method and by using of the alternative methods, as well as the difference between the values of the TWA that was obtained by comparing in the same way, were within the half of a decibel. The difference between the noise dose value obtained by the application of the SCANAM method and by the alternative method was less than $0.5 \%$. The accuracy of using the SCANAM method can be assessed as satisfactory, especially if one takes into account the long interval of time in which the measurements were performed, as well as the number of measured noise levels (2880). Accordingly, the application of the SCANAM method can be recommended for the evaluation of noise, especially when one takes into account that the application of this method is significantly cheaper than the application of separate instruments (phonometers, dosimeters, etc.), for the determination of the same values.

It is planned that the commercial version of SCANAM software be around U.S. \$ 50, depending on the version. Given that, the application of the SCANAM method is less-expensive solution than any other known solution for measuring and evaluating of noise. In addition, it does not require qualified personnel for application. However, it is not intended to replace expert analysis and specialists in this field. It is primarily designed for everyday use in environments where there are high levels of noise, or for the periodic application in conditions when changing equipment and technological process. Of course, this method can be successfully used by experts in the field of ergonomics and safety at work.

\section{Acknowledgements}

This work is supported in a part by a grant from the Ministry of Science and Technological Development, Serbia.

\section{References}

[1] C. M. Barnum, Usability testing and research, Longman, New York, 2002.

[2] E. J. McCormick and M. S. Sanders, Human factors in engineering and design, Mc GRAW-HILL, Singapore, 1984.

[3] M. S. Sanders and E. J. McCormick, Human factors in engineering and design, Mc GRAW-HILL, Singapore, 1993.

[4] M. Simonovic, D. Kalic and P. Pravica, Noise (in Serbian), Institut za dokumentaciju zastite na radu, Nis.

[5] A. Zunjic and M. Culic, Practicum for laboratory exercises in industrial ergonomics (in Serbian), University of Belgrade Faculty of Mechanical Engineering, Belgrade. 